Revista Mexicana de Economía y Finanzas, Vol. 8, No. 1, (2013), pp. 101-127

\title{
MODELO DE VALUACIÓN DE EMPRESAS ESTRATEGGICAS DESCENTRALIZADAS QUE EXPLORAN Y EXPLOTAN RECURSOS NATURALES (CASO PEMEX)
}

Dra. Araceli Espinosa Elguea*

Scotiabank Subdirectora de Análisis de Deuda Scotia Capital México

Dr. Humberto Valencia Herrera

Instituto Tecnológico de Estudios Superiores de Monterrey

Campus Ciudad de México

(Recibido 18 de junio 2012, aceptado 12 de diciembre 2012)

\section{Resumen}

La valuación de empresas descentralizadas que exploran y explotan recursos naturales (caso Pemex), interpretándolas como "Vehículo de Propósito Especial" (VPE), permite un análisis profundo y transparente, cuando la entidad no es dueña de los activos generadores de flujo operativo, tiene capital contable negativo, presenta una generación de flujo libre de efectivo negativa y está sujeta a un régimen fiscal asumido como "regalías" que no le permiten la deducibilidad de intereses. Además su elevada carga tributaria la obligan a emitir deuda para financiar su Capex. La propuesta es que estas empresas pueden allegarse de fuentes monetarias bajo cualquier esquema financiero, incluyendo capital privado, sin afectar la soberanía de los recursos naturales de cada país.

\begin{abstract}
A valuation of companies that explore and exploit natural resources (as Pemex's case), interpreting them under the optics of a "Special Purpose Vehicle" (SPV), it allows a detailed analysis and transparent, when the entity does not own the assets generating operational flow, has negative equity, presents a generation of free cash flow negative and is subject to a tax regime assumed as "royalties" that do not allow the deductibility of interest. Furthermore its high taxes oblige it to issue debt to fund its capex. The proposal is that these companies can cleave monetary sources under any financial scheme, including private equity, without affecting sovereignty of each country's natural resources.
\end{abstract}

Clasificación JEL: O13, E47 E58

Palabras clave: Recursos naturales, Costo de capital, Régimen fiscal

* Calle del Puente \#222 Col. Ejidos de Huipulco, Tlalpan C.P. 14380, México D.F. Tel. 5483 2020. E-mail:aracelielguea@prodigy.net.mx 


\section{Introducción}

Pemex al igual que otras empresas descentralizadas en el mundo, cuya actividad es la exploración y explotación ${ }^{1}$ de recursos naturales, que son estratégicos para un país, están sujetas a regímenes fiscales relacionados con pagos de regalías o derechos, que difieren en forma y en porcentaje a la renta gravada de las empresas privadas, ${ }^{2}$ impidiendo entonces la homologación en los supuestos de deducibilidad en el costo financiero de sus pasivos. Este último, es un componente del costo promedio ponderado de capital (WACC por sus siglas en inglés) necesario para calcular el valor presente de los flujos operativos futuros bajo el método de valuación tradicional DCF (Valencia-Herrera, (2009)).

Asimismo,la actual estructura financiera de Pemex presenta un deficit en la aportación de sus accionistas (Reporte de Resultados, Pemex, (2011)), y aún bajo la Reforma Energética aprobada en 2008 en México (Todo sobre la reforma energética, (2011)) que reduce marginalmente la carga fiscal a Pemex, se obtiene una corrección gradual para la reinversión de utilidades.

La decisión de valuar a Pemex como un bono estructurado emitido por un Vehículo de Propósito Especial, VPE (Fabozzi, 2000, pp. 93 y 152) se explica de la siguiente forma: la empresa no es dueña de los activos de hidrocarburos generadores del flujo operativo de Pemex, sino que el gobierno mexicano, siendo constitucionalmente (Art. 27 Constitucional, 1917) el único dueño de los mismos y sin opción a venta, concesiona a la paraestatal para explorar, explotar y comercializar petróleo y gas. Pemex paga derechos ("regalías") al gobierno a cambio de los flujos generados en su actividad y necesita emitir deuda para cubrir sus necesidades de financiamiento e inversión.

De manera similar, los VPE son entidades legales creadas únicamente para servir una función en particular, como de financiamiento o de inversión, y tienen también personalidad jurídica propia que les permite realizar la adquisición legal de activos o de flujos generados por estos activos, pagando por ellos con recursos provenientes de bonos emitidos por el propio VPE. El pago de intereses y amortizaciones de estos bonos están respaldados con los flujos que se recibirán por los activos o flujos adquiridos. Las bursatilizaciones de activos funcionan de manera similar (Fabozzi, et al. 2006).

Para valuar un bono estructurado se obtiene el valor presente de los flujos esperados originados por los activos que respaldan a la emisión, con el objeto de medir si son suficientes para cubrir la carga financiera y la amortización del mismo bono (Fabozzi (2000)). Esta misma lógica la aplicamos para Pemex, calculando el valor presente de los flujos esperados de su actividad y midiendo si son suficientes para pagar sus obligaciones financieras.

Finalmente, si valuamos los instrumentos derivados de este bono estructurado (Pemex), a través de un modelo binomial (Hull, (2008)), podemos suponer escenarios que modifican el valor de la empresa.

\footnotetext{
1 Art. 254 de la Ley Federal de Derechos

2 Tasa aplicable para personas morales se modifica de $28 \%$ a $30 \%$ para 2010,2011 y 2012 de acuerdo al Artículo Segundo de las Disposiciones de Vigencia Temporal de la Ley del Impuesto Sobre la Renta, Reforma Fiscal 2010.
} 
Este artículo se encuentra ordenado de la siguiente forma: en la sección dos se presenta el marco teórico, con una primera parte que explica la teoría de valuación tradicional de empresas y una segunda con la correspondiente a la valuación de bonos estructurados. La sección tres discute la metodología del trabajo, mientras que la sección cuatro presenta los resultados de este ejercicio. $\mathrm{El}$ apartado final contiene las conclusiones.

\section{Marco Teórico}

\subsection{Teoría de Valuación Tradicional de Empresas}

De acuerdo a Valencia-Herrera (2009) para realizar la valuación de empresas o proyectos hay tres paradigmas equivalentes entre sí: Flujos de Efectivo Descontados (FED), Flujos de Efectivo Neto a Capital Propio (FECP) y Valor Presente Ajustado (VPA).

El enfoque FED considera el costo promedio ponderado de capital y los flujos de efectivo después de impuestos. Éste es quizá el más utilizado para la valuación de una inversión de capital; para poder ser utilizado, se requiere suponer una estructura de capital constante, entendida como las proporciones de las diferentes fuentes de financiamiento que entran en el proyecto en cuestión y que deberán de mantenerse durante la vida del proyecto. Frecuentemente esta elección es adecuada si la empresa tiene una estructura de capital objetivo que no la varía en el tiempo, aunque en un momento particular es posible que no sea respetada perfectamente.

El enfoque FECP toma en cuenta el punto de vista de los accionistas (que para el caso Pemex no los hay): se consideran los flujos de efectivo a los accionistas, así como el costo de capital para ellos. Tanto los flujos de efectivo como el capital se consideran después de impuestos.

El enfoque VPA considera los flujos de efectivo de la empresa a los accionistas; tanto éstos, como el costo promedio ponderado de capital se consideran antes de impuestos. El impacto del costo de los impuestos se considera en un paso posterior.

Los tres enfoques son equivalentes entre sí cuando se cumplen tres condiciones:

1.- La estructura de capital de la empresa es constante o similar a una razón objetivo.

2.- La tasa de impuestos corporativa es constante o tiende a ser constante. No hay otros impuestos que afecten la estructura de capital.

3.- Los costos de las diferentes fuentes de capital tienden a ser constantes en el tiempo o crecen todas en la misma proporción.

Las tres condiciones anteriores, y en especial las dos primeras no son aplicables a la realidad de Pemex. La primera incluye para Pemex la existencia de un capital contable negativo5, y la segunda no aplica para Pemex por estar sujeto a un régimen fiscal enfocado a regalías. ${ }^{1}$

\footnotetext{
1 Art. 254 de la Ley Federal de Derechos
} 
104 Nueva Época REMEF (The Mexican Journal of Economics and Finance)

\subsection{Método de Flujos de Fondos Descontados (DCF por sus siglas en inglés "Discounted Cash Flow")}

Este método consiste en calcular los flujos de efectivo futuros, ajustados según su valor en el tiempo, descontados a una tasa que represente el costo de oportunidad de obtención del capital empleado (Koller et al., 2010). El valor total de la empresa se compone del valor actual de los flujos de efectivo dentro del período de pronóstico más un valor de continuación que represente los flujos de efectivo después del período del pronóstico.

La valuación de una inversión usando los flujos de efectivo de la empresa, los descuenta con costo porcentual promedio de capital después de impuestos de la empresa.

$$
\begin{aligned}
\text { Valor presente a tráves de DCF } & =\frac{\text { Flujo } t_{1}}{W A C C t_{1}}+\frac{\text { Flujo } t_{2}}{W A C C t_{2}} \\
& +\frac{\text { Flujo } t_{3}}{W A C C t_{3}}+\text { Valor de continuación }
\end{aligned}
$$

La valuación de una inversión usando los flujos de efectivo de la empresa, los descuenta con costo porcentual promedio de capital después de impuestos de la empresa.

En el caso de que la empresa tenga una política objetivo de deuda y capital, el costo de financiamiento de la inversión se puede estimar usando el costo promedio ponderado de capital después de impuestos (WACC, por sus siglas en inglés Weighted Average Cost of Capital), que consiste en estimar el porcentaje de cada una de las fuentes de financiamiento que utiliza la empresa y cuál es el costo financiero de cada una de ellas. En el caso de que la empresa tenga dos fuentes de financiamiento, deuda y capital, y los costos de capital de la deuda y el capital propio antes de impuestos sean $r_{c}$ y $r_{d}$ y la tasa marginal de impuestos corporativos sea $T$, el costo promedio ponderado de capital después de impuestos, $r$ CPDI será:

$$
\begin{aligned}
\text { Wacc } & =\left[\begin{array}{c}
\text { Capital } \\
\text { Capital + Pasivos }
\end{array} r_{c}=\text { Costo de Capital }\right] \\
& +\left[\begin{array}{c}
\text { Pasivos } \\
\text { Capital + Pasivos }
\end{array} * \quad r_{d}=\text { Costo de la Deuda (1-T) }\right]
\end{aligned}
$$

El costo del capital propio, puede ser estimado usando un modelo de valuación de activos; en el caso de utilizar el Modelo de Valuación de Activos de Capital (CAPM, por sus siglas en inglés de Capital Asset Pricing Model), el costo del capital será igual a la tasa libre de riesgo más una prima por el riesgo de la empresa, que en este modelo se estima multiplicando la Beta, $\beta$, del proyecto de inversión en cuestión, que mide el riesgo sistemático de 
la empresa, a la prima de riesgo de mercado; la diferencia entre el rendimiento de mercado y la tasa libre de riesgo, ( Briham y Houston, (1988)).

El modelo CAPM fue desarrollado por Sharpe (1964) y Litner (1965). Ambos basaron sus estudios en las investigaciones de Markowitz y Tobin (1960) quienes afirmaron que todos los inversionistas seleccionan sus carteras a través del criterio de la media-varianza. El objetivo del modelo es cuantificar e interpretar la relación que existe entre el riesgo y el rendimiento; ya que a través de esta relación lineal se puede establecer el equilibrio de los mercados financieros.

$$
\mathrm{E}\left(r_{i}\right)=\mathbf{r}_{\mathbf{f}}+\beta_{\mathbf{i m}}\left[\mathbf{E}\left(\mathbf{r}_{\mathbf{m}}\right)-\mathbf{r}_{\mathbf{f}}\right]
$$

donde:

$E\left(r_{i}\right)$ : es la tasa de rendimiento esperada de capital sobre el activo $i$.

$\beta_{\text {im }}$ : es la beta (cantidad de riesgo con respecto al Portafolio de Mercado) también conocida como:

$$
\beta_{i m=} \operatorname{Cov}\left(\mathbf{r}_{\mathbf{i}}, \mathbf{r}_{\mathbf{m}}\right) / \operatorname{Var}\left(\mathbf{r}_{\mathbf{m}}\right)
$$

$[E(r m)-r f]$ : es el exceso de rentabilidad del portafolio de mercado.

$\left(r_{m}\right)$ : Rendimiento del mercado.

$\left(r_{f}\right)$ : Rendimiento de un activo libre de riesgo.

Es importante tener presente que se trata de un Beta no apalancada, es decir que se supone que una empresa no tiene deuda en su estructura de capital, por lo tanto no se incorpora el riesgo financiero, y en caso de querer incorporarlo, debemos determinar una Beta apalancada; por lo tanto el rendimiento esperado será más alto.

Beta : Es la medida del riesgo sistemático o no diversificable de un activo. Mide la sensibilidad del valor de una acción frente a variaciones en el mercado. Un valor beta de 0.7 significa que el rendimiento de la acción o cartera probablemente aumentará o disminuirá en $70 \%$ del cambio medio del mercado. Un valor beta de 1.2 indica que el rendimiento probablemente será $20 \%$ superior o inferior al cambio medio del mercado. El indicador beta es un índice de riesgo sistemático, es decir, el riesgo en relación con las condiciones generales del mercado que no pueden modificarse.

Beta no apalancada: De acuerdo a Valencia-Herrera (2009), a ésta también se le conoce como beta de la empresa. Estrictamente la beta de la deuda a considerar debe ser la beta de la deuda después de impuestos. Dado que la beta de la deuda es relativamente pequeña, se suele suponer que es cero. En el caso de que aumente mucho el apalancamiento, la beta de la deuda puede ser que se modifique y esta relación dejará de ser válida; sólo es válida para cambios pequeños en la mezcla de deuda y capital.

Valor de continuación o Perpetuidad: Definido como el valor de la empresa más allá del horizonte de planificación, que son los flujos de fondos operativos proyectados. Este valor de continuación se puede estimar usando la relación de Gordon, siendo esta:

$$
V C_{T}=F E_{T+1} / r-g=F E_{T}(1+g) / r-g
$$


En donde,

VCT: es el valor de continuación en el año $T$,

$g$ : es la tasa de crecimiento de los flujos de efectivo a perpetuidad y

$r$ : es el costo de financiamiento.

La beta y, por tanto, el costo de capital de una empresa diferirán dependiendo del grado de apalancamiento que se tenga. Dado que las dos fuentes principales de riesgo que inciden en el costo de capital de un proyecto de inversión son el riesgo sistemático en el negocio y el riesgo derivado del apalancamiento, es común que para efectos de estimar el Costo de Capital se haga un proceso de cuatro pasos:

1.- Se estima el costo de capital accionario de empresas semejantes de las que se tenga información de mercado, esto es, el costo de capital apalancado.

2.- Se estima el costo de capital no apalancado de estas empresas.

3.- Se estima el costo de capital no apalancado del proyecto de inversión en cuestión.

4.-Se estima el costo de capital apalancado del proyecto de inversión en cuestión.

Para efectuar el paso 1 y 4 se puede trabajar ya sea con costos de capital antes de impuestos, después de impuestos o usando otra aproximación. El costo promedio ponderado después de impuestos se puede estimar usando la ponderación de deuda y capital y los costos de deuda antes de impuestos y el capital propio.

A la beta de la empresa $\beta_{E}$ también se le llama beta no apalancada. Estrictamente, la beta de la deuda a considerar debe ser la beta de la deuda después de impuestos. Dado que la beta es relativamente pequeña, se suele suponer que es cero. En el caso de que aumente mucho el apalancamiento, la beta de la deuda puede ser que se modifique, y esta relación dejará de ser válida; sólo es válida para cambios pequeños en la mezcla de deuda y capital. La beta apalancada se puede usar para estimar el costo de capital usando un modelo como el CAPM

\subsection{Teoría de Valuación de Bonos Estructurados}

De acuerdo con Fabozzi (2000) y Fabozzi, et al. (2006), el concepto de "Finanzas Estructuradas" se define como el conjunto de técnicas que se utilizan siempre que el originador o dueño de ciertos activos, que requiera cubrir necesidades de fondeo, liquidez, transferir riesgo, o alguna otra necesidad, y que no puede tener acceso o no represente una alternativa óptima, pueda emplear algún mecanismo o producto tradicional de financiamiento.

El campo de las Finanzas Estructuradas incluye las ramas de "Bursatilizaciones de activos", así como otros campos de los cuales no son objeto de este artículo, como el Arrendamiento Financiero y el Financiamiento de Proyectos.

Cualquier financiamiento estructurado, involucra los siguientes elementos:

1.- Transferencia financiera de activos o de la exposición de riesgo, con el fin de alcanzar objetivos contables, regulatorios y/o fiscales. 
2.- Transacción protegida a través de un Vehículo de Propósito Especial (VPE o Fideicomiso)

3.- La emisión de un bono que está respaldado por activos

4.- Incluye una combinación de productos derivados de tasas de interés o de crédito

Definición de Bursatilización ("Securitization") de acuerdo a Fabozzi et al. $(2006,13)$ : Obtención de liquidez a través de la transferencia de activos financieros (créditos, cuentas por cobrar, flujos generados por activos) a un "VPE o Vehículo de Propósito Especial", que a su vez emite bonos estructurados, que reciben este nombre, porque existe un activo subyacente vinculado al mismo, de donde se espera recibir flujos futuros de efectivo.

De esta forma, los activos financieros se aíslan o separan del riesgo propio de la empresa o en este caso, del país que los originó. La calidad crediticia de los activos o flujos traspasados al fideicomiso, va a soportar una gran parte de la calificación crediticia que se asigne a los bonos emitidos.

La condición más importante que exige el mercado, es que el VPE cuente con el financiamiento suficiente para completarlo y que los acreedores confíen en los flujos esperados del mismo, para garantizarles su pago. La valuación de un bono estructurado está en función de la valuación de los activos o flujos subyacentes en el instrumento.

Las características generales de los activos subyacentes o de sus flujos (colateral) y la estructura propia de este tipo de emisiones son:

El colateral de un bono estructurado puede clasificarse como "activo amortizable" o "activo no amortizable". Los primeros reciben flujos periódicos de principal y de intereses durante la vida del mismo, a través de un calendario establecido. Cualquier exceso en el pago del principal se le denomina "prepago", que puede ser total o parcial. Los "activos no amortizables" no cuentan con un calendario de pagos y solo se reciben pagos mínimos; si el pago es menor al interés, el faltante se suma al saldo del activo y si es mayor se deduce del mismo saldo.

En el caso de un "activo amortizable", la proyección de sus flujos requiere considerar a los prepagos, los cuales dependerán del nivel de tasas de interés relacionadas al activo. La proyección de los prepagos es clave para que los dueños de estos activos tomen ventaja de una baja en las tasas de interés, a niveles inferiores a la de dichos activos.

La modelación de "default" para el colateral es crítica para estimar los flujos esperados de dichos activos, así como la tasa de recuperación de los mismos en caso de impago. El cálculo de la vida promedio es otro parámetro útil para la valuación de estos bonos.

Hay elementos que ayudan a reducir el riesgo de crédito de estos bonos, que en inglés se les conoce como "credit enhancement", como pueden ser: la presencia de aforos (diferencia a positiva entre el valor de los activos y la deuda emitida por el VPE), seguros crediticios, reservas, estructuras que contemplan emisiones de bonos senior y subordinados, donde las primeras están respaldadas por activos de mejor calidad crediticia y las segundas con mayor riesgo de crédito. 


\section{Nueva Época REMEF (The Mexican Journal of Economics and Finance)}

Algunas veces los bonos incluyen cláusulas de "llamados anticipados" para poder ser amortizados anticipadamente en una fecha determinada.

\subsection{Valuación de bonos estructurados}

El modelo a usar para valuar este tipo de bonos depende de las características del activo y de los flujos que respalden a la emisión (Fabozzi, (2000)). Hay dos aproximaciones para valuarlos:

1.- Z-spread (spread con volatilidad nula). Es una medida del diferencial en tasas (spread) que el inversionista le gustaría obtener sobre la curva de tasas spot (tasa de cotización de mercado del día de la valuación), en caso de que el bono se mantenga hasta el vencimiento. Aquí se utiliza la tasa de interés spot + un diferencial o "spread" para descontar los flujos esperados. El valor del bono es entonces el valor presente de dichos flujos, descontados a esta tasa. Este tipo de valuación puede aplicarse a bonos que no tienen opciones (como de prepago), o que las tienen pero los dueños de los activos bursatilizados no tienen una clara tendencia a ejercerlas (Fabozzi, (2000)).

Ya que el spread que va a hacer que el valor presente de los flujos futuros sea igual al valor de mercado cuando se descuenten a una tasa equivalente a "tasa spot + spread", entonces se requiere de un procedimiento de prueba y error para encontrar el z-spread.

2.- Spread ajustado por opciones (OAS por las siglas en inglés de Option-Adjusted Spread). Se utiliza para valuar bonos con opciones. En este caso puede utilizarse un Modelo Binomial 13 o el Modelo de Simulación Montecarlo (Hull, (2000)).

En el Modelo Montecarlo, el OAS es el diferencial en tasas o spread, que cuando se agrega a una tasa de interés spot en todas las posibles simulaciones, hace que el valor presente promedio de todos los escenarios sea igual al precio de mercado observado (más intereses devengados). Matemáticamente el OAS es el valor para $K$ (el spread) que satisface la siguiente condición:

$$
\begin{aligned}
& \mathrm{VP}(\text { Escenario } 1)+\mathrm{VP}(\text { Escenario } 2)+\cdots+\mathrm{VP}(\text { Escenario N }) / \mathrm{N} \\
& =\text { Precio de Mercado }
\end{aligned}
$$

donde : $N$ : es el número de escenarios de tasa de interés.

El objetivo es determinar a qué spread $K$, el modelo arrojará un valor teórico igual al precio de mercado. El procedimiento para determinar el OAS es sencillo e involucra la misma búsqueda de algoritmo que se aplica para el Z-spread.

El OAS se utiliza para reconciliar el valor teórico con el precio de mercado. El valor presente promedio de todos los posibles escenarios son los datos que alimentan al modelo, y que se le conoce como valor teórico. Lo que busca un inversionista es comprar un bono donde su valor teórico sea mayor al precio de mercado. Pero además el modelo convierte la divergencia entre el precio de mercado y el valor teórico en una medida de spread, dado que la mayoría de los participantes de los mercados están acostumbrados a tomar decisiones con spreads. 
Este spread se compara con tasas de referencia, que al mismo tiempo fueron utilizadas para la construcción de los diferentes escenarios. Esta medida es una "opción ajustada" ya que los flujos descontados bajo diferentes escenarios de tasas son ajustados por la opción de prepago.

El Modelo Binomial. En el caso de bonos que incluyen opciones, es necesario tomar en cuenta la volatilidad de las tasas de interés. Esto se puede hacer a través de un árbol binomial de tasas de interés. Se trata de solo una descripción gráfica de un periodo o de tasas de interés de corto plazo a través del tiempo bajo algún supuesto de la volatilidad de las tasas de interés.

La gráfica siguiente es un ejemplo de árbol binomial para tasas de interés. En este árbol, cada nodo (cada punto) representa un periodo de tiempo, que puede ser por ejemplo un año desde el nodo hacia la derecha. Cada nodo se etiqueta con la letra $N$. Existen "subletras" (utilizaremos siglas de palabras en inglés), excepto para el primer nodo, donde $L$ significa la menor de dos tasas en un periodo y $H$ representa la mayor de dos tasas de interés en un periodo. Por ejemplo el nodo $N_{H H}$ significa que para poder llegar a este nodo se necesita seguir la siguiente ruta: en el primer periodo hacia la mayor de las dos tasas y en el segundo periodo nuevamente seguir hacia la mayor de dos tasas.

Figura 1. Árbol Binomial de Tasas de Interés

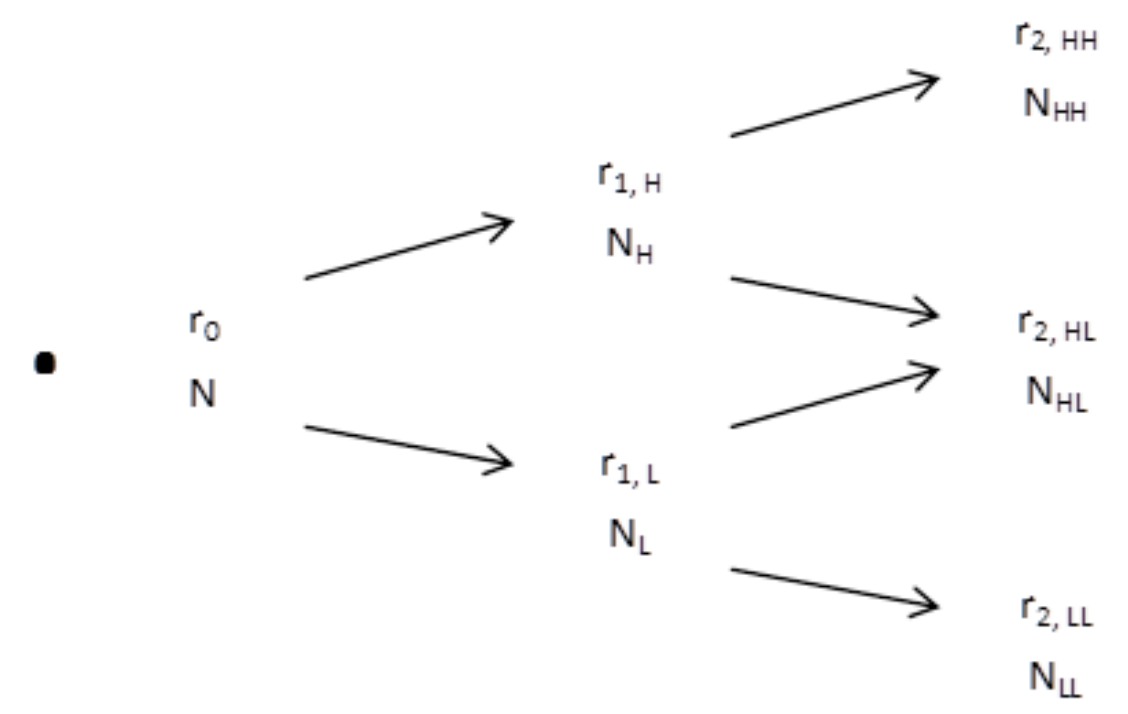

El punto negro, denotado con $N$ es la raíz del árbol y se refiere a la actual tasa de interés spot denotada por $r_{0}$. La idea que existe al crear este árbol es que la tasa de interés a un año puede tomar dos posibles valores en el siguiente periodo y las dos tasas tienen la misma probabilidad de ocurrir. Una tasa será más alta que la otra.

Se utiliza la siguiente notación para describir el árbol en el año 1:

$\delta_{t}=$ volatilidad asumida para la tasa de interés a 1 año 
$r_{1, L}=$ la menor de la tasa a 1 año para el siguiente periodo

$r_{1, H}=$ la mayor de la tasa a 1 año para el siguiente periodo

La relación entre $r_{1}, L$ y $r_{1}, H$ es:

$$
r_{1, H}=r_{1, L}\left(e^{2 \delta}\right)
$$

En el segundo año hay tres posibles valores para la tasa a 1 año $r_{2, L L}=$ la tasa de interés en el segundo periodo, asumiendo la menor tasa en el año uno y en el año dos $r_{2, H H}=$ la tasa de interés en el segundo periodo, asumiendo la mayor tasa en el año uno y en el año dos $r_{2, H L}=$ la tasa de interés en el segundo periodo, asumiendo la mayor de la tasa en el año 1 y la menor en el año 2 La relación entre $r_{2, L L}$ y las otras dos tasas es como sigue:

$$
r_{2, H H}=r_{2, L L}\left(e^{2 \delta}\right) \mathrm{y} r_{2, H L}=r_{2, L L}\left(e^{2 \delta}\right)
$$

Las tasas de interés del árbol binomial son tasas forward. Cuando se valúan bonos con opciones se utilizan conjuntos de tasas forwards. De acuerdo a Frank Fabozzi (2000), bajo ciertos supuestos, las tasas forward pueden ser vistas por el consenso del mercado como las tasas de interés futuras. Las tasas forward pueden obtenerse de la curva teórica de tasas spot de bonos gubernamentales, a través de una extrapolación implícita, por lo que también se les denomina "tasas forward implícitas".

\subsection{Determinando el valor de un nodo}

Para encontrar el valor del bono en un nodo, primero calculamos el valor del bono a la mayor tasa y el valor del bono a la menor tasa, del nodo que se encuentra a la derecha. Por ejemplo para el nodo $N_{H}$ se necesita primero determinar el valor de $N_{H H}$ y de $N_{H L}$

Las opciones se valúan empezando desde el final del árbol (tiempo T) y trabajando desde el final hacia el principio del árbol. El valor de la opción es conocido en el momento $T$.

En términos generales, el valor de un bono en un determinado nodo sigue la regla básica para valuación: el valor presente de los flujos esperados. Dado que hay dos valores presentes: el valor presente si la tasa a un año es la mayor o si la tasa es la menor. Entonces si suponemos que la probabilidad de que ambas ocurra es igual, y entonces obtendríamos un promedio de los dos valores presentes.

\section{Metodología}

La aplicación práctica del Método de Flujos Descontados, se realizó con base en la metodología propuesta por en Valencia-Herrera (2009), siguiendo el siguiente proceso:

\subsection{Investigación}

Para entender Pemex, se requirió de una profunda labor de Investigación sobre la historia y situación actual de la empresa, y con ello contar con bases sólidas para establecer supuestos para la proyección de 10 años de los principales estados financieros: balance, resultados y flujo de efectivo. ${ }^{3}$

\footnotetext{
3 Supuestos y modelos de estimación de balance, resultados y flujo de efectivo de Pemex, están disponibles en archivos en Excel. Solicitar al correo: aracelielguea@prodigy.net.mx
} 
Los puntos más importantes que destacan de esta investigación son:

a) Reservas Petroleras.- De acuerdo con el Art. 27 de la Constitución Política de los Estados Unidos Mexicanos y con la Ley Reglamentaria de Pemex, todo el petróleo, así como todas las reservas de hidrocarburos dentro de México son propiedad de la Nación. nicamente Pemex y sus Organismos Subsidiarios tienen derecho exclusivo de extraer y vender la producción de éstas reservas y no son de su propiedad.

Debido a que se trata de recursos naturales no renovables, es un reto para Pemex el incrementar la "tasa de restitución" de reservas probadas. Es decir que por cada barril de petróleo que se extraiga del subsuelo este debe ser reemplazado en forma paralela, para mantener el negocio en marcha en el largo plazo y al mismo tiempo pueda mantener su valor como empresa. Actualmente la "tasa de restitución" de reservas probadas es de $101 \%$ (Tasa de restitución(2012)).

Pemex utiliza el método contable de "Esfuerzos Exitosos" para dar un reconocimiento monetario a las inversiones incurridas que soportan el valor de las reservas. A través de este método contable, Pemex reconoce en resultados los costos y gastos, cuando se ha tenido éxito en el desarrollo de reservas, y capitaliza aquellos relacionados con reservas aún probables.

En un escenario conservador, los geólogos de Pemex esperan que la producción de petróleo para los siguientes 5 años (2010-2015) se mantendrá en niveles promedio de 2.5 millones de barriles diarios (Kogan, et al., (2011)). Pemex definió una serie de proyectos denominados estratégicos destacando el Proyecto Aceite Terciario del Golfo (antes Paleocanal de Chicontepec) y el Proyecto Aguas Profundas del Golfo (Proyectos Estratégicos, (2011)).

Figura 2. Reservas petroleras y tasa de restitución de Pemex

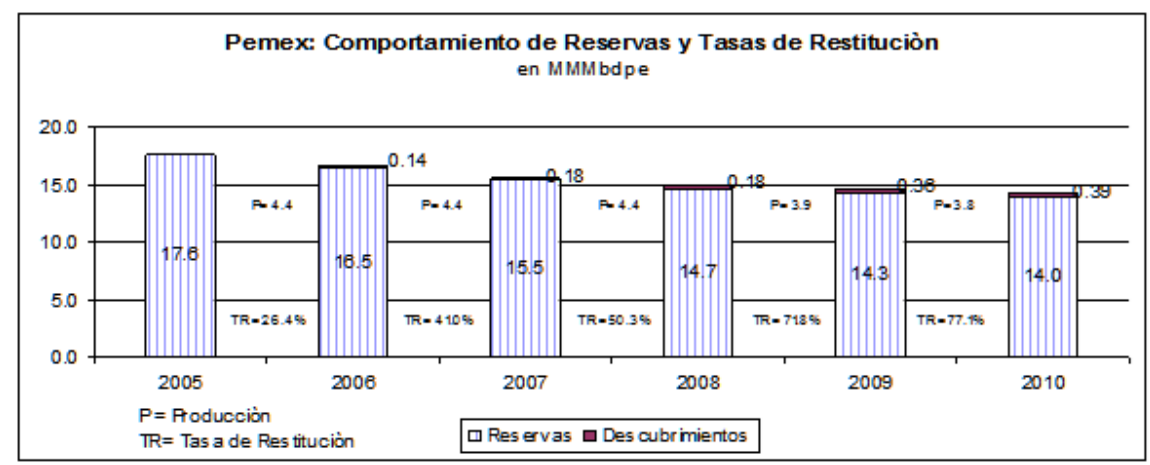

MMMbpce $=$ Miles de millones de petróleo crudo equivalente.

b) Principales Competidores.-

Pemex ocupa el segundo lugar en activos y en generación anual de ingresos entre sus competidores en América Latina, según cifras al cierre de 2009.

El nivel de apalancamiento de Pemex de 108\% (pasivos totales a activos totales) es el más alto de la región. El alto apalancamiento de Pemex está relacionado con la alta carga tributaria de la paraestatal mexicana; de acuerdo 


\section{Nueva Época REMEF (The Mexican Journal of Economics and Finance)}

al cuadro que aparece a continuación, la relación de impuestos y derechos sobre utilidad de operación, al que para efecto de este artículo se le denomina REGALÍAS, se ubicó en $108 \%$ al cierre de 2010, que es la tasa impositiva más alta entre sus competidores, incluyendo a empresas como Shell y Exxon.

La salida de flujo de efectivo hacia el erario público, no le deja recursos a Pemex para auto inversión, sino que forzosamente debe recurrir a fuentes de financiamiento externas, para mantener su crecimiento. En 2010 los intereses pagados netos, por concepto de pasivos ascendió a U\$5,887 millones (MXN $\$ 74,382$ millones). En cuanto a nivel de reservas probadas Pemex ocupa el lugar número 17 en el mundo, aportando $0.86 \%$ de las reservas mundiales.

Figura 3. Pemex y sus Competidores Globales: Comparativo de Impuestos, Derechos - Regalías sobre Hidrocarburos. Millones de dólares, excepto Ecopetrol.

\begin{tabular}{|c|c|c|c|c|c|c|c|}
\hline & \begin{tabular}{|l|} 
PEMEX \\
(1)
\end{tabular} & $\frac{\text { PDVSA }}{\text { (1) }}$ & $\frac{\text { ECOPET }}{\frac{\text { ROL }}{\text { (1) }}}$ & \begin{tabular}{|c|} 
PETROBRA \\
$\underline{s}$ \\
(1)
\end{tabular} & $\frac{\text { EXXON }}{111}$ & $\frac{\text { SHELL }}{\text { (1) }}$ & $\begin{array}{l}\frac{\text { BRITISH }}{\text { PETROLE }} \\
\frac{\text { UM }}{(2)}\end{array}$ \\
\hline Ventas netas & $\begin{array}{c}103,75 \\
1\end{array}$ & 94,929 & 21,927 & 91,869 & $\begin{array}{c}383,22 \\
1\end{array}$ & $\begin{array}{c}388,05 \\
6\end{array}$ & 239,272 \\
\hline $\begin{array}{l}\text { Utilidad antes de } \\
\text { derechos e impues tos } \\
\text { sobre hidrocar buros }\end{array}$ & 49,096 & 20,455 & 6,004 & 22,061 & 52,959 & 21,020 & 26,426 \\
\hline $\begin{array}{l}\text { Derechos e impuestos } \\
\text { sobre hidrocar buros }\end{array}$ & 52,936 & 12,884 & 3,341 & 5,238 & 21,561 & 8,302 & 8,365 \\
\hline $\begin{array}{l}\text { Derechos e impuestos } \\
\text { / Utilidad antes de } \\
\text { derechos e impuestos } \\
\text { s/hidrocar buros }\end{array}$ & $108 \%$ & $63 \%$ & $58 \%$ & $24 \%$ & $54 \%$ & $40 \%$ & $32 \%$ \\
\hline $\begin{array}{l}\text { Derechos e impuestos } \\
\text { / Ventas }\end{array}$ & $51 \%$ & $14 \%$ & $15 \%$ & $6 \%$ & $17 \%$ & $3 \%$ & $4 \%$ \\
\hline
\end{tabular}

(1) Cifras al 31 de diciembre de 2010, (2) cifras al 31 de diciembre 2009, ya que en 2010 reporta pérdidas antes de impuestos, por derrame petrolero en Golfo de México. Fuente: Informes anuales de las empresas seleccionadas.

c) Pasivos Laborales.- El monto de pasivos laborales al 31 de diciembre de 2010 fue de $\$ 661,365$ millones de pesos (US\$ 52,332 millones) cifra superior en $\$ 85,164$ millones de pesos (US\$ 6,739 millones) a la reportada al cierre de 2009. El número total de empleados al cierre de 2009 fue de 145,146 de los cuales aproximadamente el $71 \%(103,054)$ son sindicalizados y el resto de confianza (Reporte de Resultados, (2011)).

Los pasivos laborales pueden representar un riesgo de liquidez futuro para Pemex, dependiendo del nivel en el que se ubique el precio de venta del petróleo. En 2010 se erogaron en efectivo US\$2,228 millones (MXN\$28,161 millones) por concepto de "Contribuciones y Pagos por Beneficios a Empleados".

Bajo reglas contables internacionales, ${ }^{4}$ todos los pasivos laborales registrados con anterioridad a 2008 deberán ser amortizados en un periodo

\footnotetext{
4 Ver Estados Financieros Auditados al 31 de Diciembre de 2010, pp. 16, y 29, Nota 12. / FAS (2010).
} 
de 5 años. Y a partir de 2009, los beneficios laborales que participaron en la generación de ingresos del ejercicio, son reconocidos directamente en resultados.

Pemex contabiliza los pasivos laborales bajo la modalidad de "Obligación por Beneficios Definidos", 5 lo que significa que la empresa es la responsable de este pasivo en su totalidad, así como el de fondear los recursos para creación de reservas y el cuidar el adecuado manejo de estos recursos, para que no pierdan valor a través del tiempo.

En el cálculo del valor presente de las obligaciones laborales bajo la modalidad de "Obligación por Beneficios Definidos" (OBD), se consideran los pagos probables con independencia de si existirán o no aportaciones al plan. Se calcula con base en los servicios prestados con sueldos proyectados o en el costo futuro, tanto de otros beneficios al retiro como de beneficios por terminación por causas distintas a la reestructuración, en los términos de cada plan, a una fecha determinada.

En la determinación de la obligación por beneficios al retiro se incluyen a todos los empleados (jubilados y activos); además, en otros beneficios al retiro, se incluyen a empleados activos en condiciones de elegibilidad y a quienes aún no han alcanzado dicha condición de elegibilidad. En el caso de beneficios por terminación por causas distintas a la reestructuración se incluyen a todos los empleados en activo.

d) Reforma Energética 2008.- Incluye siete decretos, aquí solo se comentan los más relevantes y que fueron recopilados del documento "Todo sobre la Reforma Energética" publicado por Pemex en la página www.pemex.com.

- Primer Decreto: Modificaciones a la Ley de Pemex

Nueva Estructura de Gobierno: Se integran 4 consejeros profesionales.

Creación de Comités Ejecutivos: Auditoría y Evaluación del desempeño; Transparencia; Remuneración; Estrategia e Inversiones; Adquisiciones y Arrendamientos; Medio Ambiente y Desarrollo e Investigación Tecnológica.

Esquema de Contratación para Actividades Sustantivas (Comité de Adquisiciones y Arrendamientos). Contratos Integrales de Servicios para Exploración y Producción (antes Contratos Incentivados).

Compensaciones en efectivo a los contratistas que provean beneficios de avances tecnológicos, ejecución y/o rendimiento.

No se compartirán los derechos de propiedad de los hidrocarburos.

Penalización por incumplir metas o cometer infracciones ambientales.

Cambios en el Régimen Fiscal de Pemex. En Octubre de 2009, se aprobó el dictamen de la Ley Federal de Derechos en materia del régimen fiscal de PEP. El dictamen contempla cambios en los regímenes de Chicontepec y para Aguas Profundas:

- Derecho Especial: Se reduce a 30\% la tasa del Derecho Especial aplicable sobre el valor de la producción menos deducciones autorizadas. Esta tasa aumentará a $36 \%$ cuando la producción acumulada del campo de que se trate supere los $240 \mathrm{MM}$ de barriles de petróleo crudo equivalente. (bpce). El

\footnotetext{
5 Ver IAS 19
} 


\section{Nueva Época REMEF (The Mexican Journal of Economics and Finance)}

límite de deducibilidad aplicable al cálculo del Derecho Especial aumenta para situarse en el valor mínimo entre US $\$ 32.5$ por bpce y $60 \%$ del precio del bpce. En este mismo sentido, se extiende a 15 años el período de recuperación de los costos que rebasen el límite de deducibilidad.

- Derecho sobre Extracción: Se modifica la tasa del Derecho sobre extracción de (i) una tasa variable de entre $10 \%$ y $20 \%$, en función del precio del crudo, aplicable sobre el valor de la producción, a (ii) una tasa fija de $15 \%$ aplicable sobre la totalidad de los ingresos.

- Derecho Adicional sobre Hidrocarburos: Se crea el Derecho Adicional sobre Hidrocarburos, aplicable únicamente cuando el precio del crudo equivalente supere los US $\$ 60$ por bpce. Este derecho es equivalente a una tasa de $52 \%$ sobre el volumen de producción, multiplicado por la diferencia entre el precio de referencia y US\$60.

- Segundo Decreto: Ley de la Comisión Nacional de Hidrocarburos.

Creación de la Comisión Nacional de Hidrocarburos (órgano desconcentrado de la Secretaría de Energía), con 5 miembros, que regulará y supervisará:

La exploración y extracción de hidrocarburos. Las actividades de proceso, transporte y almacenamiento relacionadas directamente con proyectos de exploración y extracción.

e) Régimen Tributario.- Los derechos que aplican a Pemex son:

- Derechos que aplican a PEP (Pemex Exploración y Producción): A partir de 2008, el régimen fiscal para PEP consiste en los siguientes DERECHOS (se mencionan los principales):

- Derecho Ordinario sobre Hidrocarburos: Aplica al valor de la producción total de petróleo crudo y gas natural extraídos en el año menos las deducciones permitidas (inversiones específicas, ciertos gastos y costos y otros derechos) .En 2009 la tasa fue de $73.5 \%$. En 2010 será de $73 \%$, 72.5\% en 2011 y $71.5 \%$ en 2012. La deducción de los costos no deberá exceder US $\$ 6.50$ por barril de petróleo y de US\$2.70 por mil pies cúbicos de gas natural no asociado.

Para el caso de los campos en el Paleocanal de Chicontepec y Aguas Profundas en el Golfo de México, a partir del $1^{a}$ de enero de 2010 este Derecho NO es aplicado al valor del petróleo y gas natural extraídos de éstos, ya que están sujetos al Derecho de Extracción de Hidrocarburos, al Derecho Especial sobre Hidrocarburos para campos en el Paleocanal de Chicontepec y el Derecho sobre hidrocarburos para Aguas Profundas.

- Derecho sobre Hidrocarburos para el Fondo de Estabilización: Este aplica cuando en el año, el precio promedio ponderado del barril de petróleo crudo exportado exceda de US\$22.00. La tasa aplicable será del $1 \%$ al $10 \%$ dependiendo del precio promedio con un tope de US\$31.00, precio a partir del cual se pagará una tasa de $10 \%$.

- Derecho para la Investigación Científica y Tecnológica en Materia de Energía: Durante 2010 se aplicará una tasa de $0.40 \%$ al valor de la producción de petróleo crudo y gas natural extraídos, para 2011 de $0.50 \%$ y de $0.65 \%$ para 2012. 
- Derecho para la Fiscalización Petrolera: La tasa es del $0.003 \%$ al valor de la producción de petróleo crudo y gas natural extraído en el año.

- Derecho Extraordinario sobre la Exportación de petróleo crudo: Se aplica una tasa de $13.1 \%$ sobre el valor de: "Multiplicar la diferencia que exista entre el precio promedio ponderado anual del barril de petróleo crudo mexicano y el precio de petróleo crudo presupuestado por el volumen anual de exportación".

- Derecho nico sobre Hidrocarburos: En éste aplica una tasa flotante anual al valor del petróleo crudo y gas natural extraído de los pozos abandonados. Esta tasa fluctúa entre $37.0 \%$ y $57.0 \%$ dependiendo del precio promedio ponderado de exportación del petróleo crudo mexicano.

Vale la pena comentar que algunos derechos pagados por Petróleos Mexicanos no tienen impacto en el flujo de efectivo de la empresa debido a que son acreditados con otros Derechos e Impuestos o son deducidos de la base impositiva de otros derechos:

- Derecho Especial sobre Hidrocarburos: Tasa del 30\% al 36\% al valor anual de petróleo crudo y gas natural extraído de los campos en el Paleocanal de Chicontepec, menos ciertas deducciones permitidas (tales como inversiones específicas, algunos gastos y costos, con ciertas condiciones).

- Derecho sobre la Extracción de Hidrocarburos: Tasa anual del 15\% al valor anual del petróleo crudo y gas natural extraídos de los campos en el Paleocanal de Chicontepec y de campos en Agua Profundas.

- Derecho Adicional sobre Hidrocarburos: Tasa del $52 \%$ a la diferencia entre el valor anual de petróleo crudo en el campo de que se trate y US $\$ 60.00$ por el volumen extraído. Este derecho se aplica únicamente a los campos localizados en el Paleocanal de Chicontepec y a los campos en aguas profundas, siempre y cuando el precio del barril de petróleo extraído sea mayor de US $\$ 60$.

Impuestos para el resto de subsidiarias de Pemex:

Impuestos a los Rendimientos Petroleros.- Se calcula aplicando al Rendimiento Neto una tasa del 30\%, según la Ley de Ingresos de la Federación, para el año fiscal correspondiente.

IEPS (Impuesto Especial sobre Producción y Servicios).- Es un impuesto indirecto sobre las ventas internas de gasolinas y diesel que Pemex Refinación recauda en representación del Gobierno Federal. El IEPS sobre la venta de gasolinas y diesel es equivalente a la diferencia entre el precio de referencia internacional de cada producto (ajustado por costos de flete, manejo y factor de calidad) y el precio de menudeo del producto a sus clientes (sin incluir el IVA, el margen comercial y los costos de flete). De este modo, el Gobierno Federal se asegura de que Pemex conserve una cantidad que refleje los precios internacionales de estos productos, mientras el Gobierno Federal se allega la diferencia entre los precios internacionales y los precios a los cuales estos productos se venden en México. La Ley de Ingresos de la Federación de 2006 a 2009 establece que los montos que resulten de las tasas del IEPS negativo pueden acreditarse contra el IEPS a cargo y si hubiera remanente, se podrá acreditar contra el IVA y si existiese todavía excedente, contra el Derecho Ordinario sobre Hidrocarburos. En caso de un IEPS negativo para el año 2010, sería acreditado de esa forma. 
ISR (Impuesto sobre la Renta). Petróleos Mexicanos y los Organismos Subsidiarios están exentos del pago del Impuesto sobre la Renta, sin embargo, algunas de la Compañías Subsidiarias son empresas mexicanas y están obligadas a cumplir con el régimen fiscal que aplica a personas morales (determinado a la tasa del $29 \%$ aplicable a los ingresos menos ciertas deducciones).

A partir del 2008 entró en vigor un nuevo impuesto corporativo (Impuesto Empresarial a Tasa nica, IETU) que impone un impuesto mínimo del $17.5 \%$ de los ingresos por ventas de la persona moral (menos ciertas deducciones y ciertos gastos de la inversión). Este impuesto ha pasado del $16.0 \%$ en 2008 a $17.5 \%$ en 2010 y subsecuentes.

Las Compañías Subsidiarias de Petróleos Mexicanos en el extranjero, pueden estar sujetas a impuestos en la jurisdicción de su residencia u operaciones.

f) Fuentes de Financiamiento

Los pasivos financieros con costo de corto y largo plazo de Pemex al cierre de 2010 alcanzaron los MXN\$665 miles de millones (US $\$ 48.4$ miles de millones), (Reporte de Resultados, (2011)).

\subsection{Cálculo del Valor Presente del Flujo Libre de la Empresa.}

Existen estados financieros proyectados y los supuestos de las proyecciones en versión electrónica de Excel.

1.- Proyección de los Estados Financieros (Balance General y Estado de Resultados).

2.- Determinación de los Flujos Libres de Efectivo (Proyección del Estado de Flujo de Efectivo).

3.- Cálculo de la Tasa del Costo de Capital (WACC).

4.- Estimación del Valor Terminal o de Continuación (Perpetuidad).

5.- Cálculo del Valor del Activo e interpretación de Resultados.

La aplicación de los cinco pasos anteriores llevó a obtener un valor de Pemex que se denominó Escenario Base. Asimismo, se llevó a cabo un Análisis de Sensibilidad, sometiendo a Pemex a cambios en tres variables y se desprendieron tres escenarios más:

Escenario Uno: Suponiendo que los pasivos laborales se eliminan, respecto al escenario base, debido a un decreto presidencial teórico.

Escenario Dos: Suponiendo que las regalías al gobierno, fueran la mitad que las que se pagan bajo el escenario base.

Escenario Tres: Determinación de un precio del petróleo que provoca una situación de equilibrio en la generación de Flujo Neto Operativo. Ejemplo cuando el precio internacional del petróleo es de US\$52.52 dólares por barril.

La aplicación práctica del Modelo Binomial, para la valuación de un bono estructurado, los flujos libres de Pemex por el periodo 2011-2020 son los que se descuentan a tasas forwards. 


\section{Resultados del Ejercicio Propuesto:}

\section{1.) Modelo de Flujos Descontados:}

La aplicación de la metodología señalada en el apartado anterior arrojó las siguientes cifras. El modelo para el cálculo de los escenarios está disponible en hojas electrónicas de Excel.

Escenario Base.- Situación actual de Pemex bajo las condiciones vigentes tanto operativas como fiscales destacando el hecho que el Capital Social de la empresa es negativo.

\section{Resultado de la Valuaciòn Escen ario Base}

\begin{tabular}{|lrr|}
\hline \hline & Mill. Pesos & Mill. Dòlares \\
\hline \hline Suma de Flujos Descontados & 962,763 & 75,879 \\
Perpetuidad Descontada & 455,069 & 35,866 \\
Valor de los activos & $\mathbf{1 , 4 1 7 , 8 3 2}$ & $\mathbf{1 1 1 , 7 4 4}$ \\
Deuda con Co sto al 30 sep. 2010 & 652,828 & $\mathbf{5 1 , 4 5 2}$ \\
Valor de la empresa & $\mathbf{7 6 5 , 0 0 4}$ \\
Valor de la empresa / Deuda con Costo & 1.17 \\
Apalancamiento (deuda con costo / Valor de la empresa) & 0.85 \\
Tipo de cambio peso / dólar al 30 Sep. 2010 & \multicolumn{2}{|c|}{12.6882} \\
\hline
\end{tabular}

Bajo estas premisas y descontando los flujos según los cuadros que enseguida se presentan, el valor neto de la empresa se ubicó en $\$ 765,004$ millones de pesos con lo que Pemex cubre 1.2 veces sus pasivos con costo. La razón de apalancamiento se ubicó en $0.85 \%$.

Cuadros A, B y C: Cálculos del Costo de Capital (Ks), WACC y flujos descontados del Escenario Base:

Cuadro A. Cálculo del $K_{s}$ a tráves del CAPM

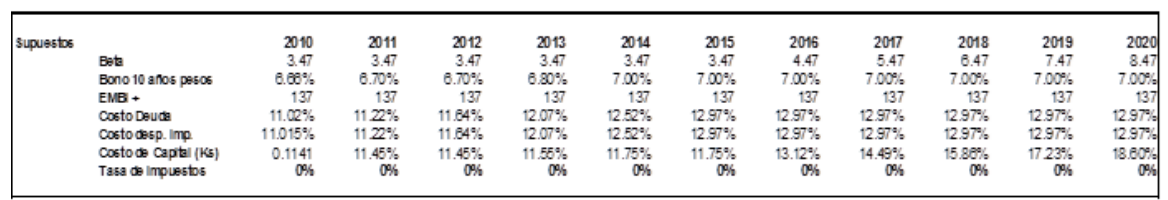

Cuadro B. Cálculo de la Tasa de Descuento

\begin{tabular}{|c|c|c|c|c|c|c|c|c|c|c|c|c|}
\hline & 2009 & 2010 & 2011 & 2012 & 2013 & 2014 & 2015 & 2016 & 2017 & 2018 & 2019 & 2020 \\
\hline Deuda con cos bo & 631,859 & 690829 & 789,977 & 884,038 & 970,927 & $1,061,056$ & $1,157,179$ & $1,239,682$ & 1,323077 & $1,407,372$ & 1,492578 & 1,578705 \\
\hline Capital & -66.840 & -101.965 & $-170,434$ & -209629 & 216831 & $-229,421$ & 222497 & $-204,192$ & $-155,159$ & $-143,814$ & -128783 & -122323 \\
\hline Deuda Malor de la empress & $112 \%$ & $117 \%$ & $128 \%$ & $131 \%$ & $129 \%$ & $128 \%$ & $124 \%$ & $120 \%$ & $113 \%$ & $111 \%$ & $109 \%$ & $108 \%$ \\
\hline Copta $/$ Natr de bempess & $-11.83 \%$ & $-17.32 \%$ & $-27.51 \%$ & $-31.08 \%$ & $-2875 \%$ & $-27.56 \%$ & $-2380 \%$ & $-19.72 \%$ & $-13.29 \%$ & $-11.39 \%$ & -9.44\% & $-840 \%$ \\
\hline WACC & $10.97 \%$ & $10.95 \%$ & $11.16 \%$ & $11.70 \%$ & $1222 \%$ & $1274 \%$ & $1325 \%$ & $1203 \%$ & $1276 \%$ & $1264 \%$ & $1250 \%$ & $1249 \%$ \\
\hline
\end{tabular}

Cuadro C. Cálculo de Flujos Descontados

\begin{tabular}{|c|c|c|c|c|c|c|c|c|c|c|c|c|}
\hline Flujos a desconter & $\begin{array}{r}2010 \\
175,482\end{array}$ & $\begin{array}{r}2011 \\
151.972\end{array}$ & $\begin{array}{r}2012 \\
137,307\end{array}$ & $\begin{array}{r}2013 \\
163.569\end{array}$ & $\begin{array}{r}2014 \\
166,707\end{array}$ & $\begin{array}{r}2015 \\
189.321\end{array}$ & $\begin{array}{r}2016 \\
201.304\end{array}$ & $\begin{array}{r}2017 \\
235.764\end{array}$ & $\begin{array}{r}2018 \\
203.140\end{array}$ & $\begin{array}{r}2019 \\
212073\end{array}$ & $\begin{array}{r}2020 \\
208936\end{array}$ & $\begin{array}{r}\text { Perpetid ad } \\
2.026 .382\end{array}$ \\
\hline 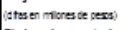 & & & & & & & & & & & & \\
\hline $\begin{array}{l}\text { Flujos des contados } \\
\text { (itsen noveser veras }\end{array}$ & 158.168 & 122988 & 98523 & 103.123 & 91,548 & 89.717 & 84,232 & 87,106 & 66.269 & 61,087 & 53,140 & 456,089 \\
\hline
\end{tabular}

Escenario Uno: Suponiendo que los pasivos laborales se eliminan, respecto al escenario base, debido a un decreto presidencial teórico. 


\section{Resultado de la valuación: Escenario sin pasivo laboral}

\begin{tabular}{|l|rr|}
\hline \hline & Mill. Pesos & Mill. Dólares \\
\hline \hline Suma de Flujos Descontados & $1,080,523$ & 85,160 \\
Perpetuidad Descontada & 756,403 & 59,615 \\
Valor de los activos & $\mathbf{1 , 8 3 6 , 9 2 6}$ & $\mathbf{1 4 4 , 7 7 4}$ \\
Deuda con Costo al 30 sep. 2010 & $\mathbf{6 5 2 , 8 2 8}$ & $\mathbf{5 1 , 4 5 2}$ \\
Valor de la empresa & $\mathbf{1 , 1 8 4 , 0 9 8}$ & \\
Valor de la empresa / Deuda con Costo & 1.81 & \\
Apalancamiento (deuda con costo / Valor de la empresa) & 0.55 & \\
Tipo de cam bio peso / dólar al 30 Sep. 2010 & & 12.6882 \\
\hline
\end{tabular}

Al sensibilizar el escenario base bajo este supuesto, se observa que el valor de la empresa es $54 \%$ superior al obtenido en el escenario base ubicándose en $\$ 1,184,198$ millones de pesos. Se atribuye esto, a que el flujo neto operativo crece en promedio 3 veces respecto al escenario base siendo esto resultado de la eliminación del costo de la reserva laboral que corresponde a jubilados y en la que existe una salida real de efectivo (en el costo de venta y la parte que se incluye en los gastos generales) principalmente.

Con el exceso de liquidez que se obtiene en este escenario, se decidió disminuir el porcentaje del financiamiento externo (bancario y bursátil) y de este modo abaratar y disminuir el monto de pasivos con costo, con los siguientes beneficios: disminución de pago de intereses, una mejor razón de apalancamiento y por tanto una mejor cobertura de deuda con costo mejorándose ambos indicadores respecto a los obtenidos en el escenario base. Asimismo, incrementé el monto de las amortizaciones de deuda. Si bien esperaba una "mejor valuación", ésta se ve afectada por la tasa de descuento que se incrementa casi en el doble (ver cuadros siguientes) respecto al escenario base y esto es debido a una mayor ponderación del capital; vale la pena destacar que bajo este escenario el capital contable deja de ser negativo a partir del primer año de estimaciones.

Cuadros D, E y F: Cálculos del Costo de Capital (Ks), WACC y flujos descontados del Escenario Uno:

Cuadro D. Cálculo del $K_{s}$ a tráves del CAPM

\begin{tabular}{|c|c|c|c|c|c|c|c|c|c|c|c|c|}
\hline \multirow[t]{8}{*}{ Supuestor } & & 2010 & 2011 & 2012 & 2013 & 2014 & 2015 & 2016 & 2017 & 2018 & 2019 & 2020 \\
\hline & & 3.47 & 3.47 & 3.47 & 3.47 & 3.47 & 3.47 & 4.47 & 5.47 & 6.47 & 7.47 & 8.47 \\
\hline & Bono 10 anos pessos & $6.60 \%$ & $6.70 \%$ & $6.70 \%$ & $6.80 \%$ & $7.00 \%$ & $7.00 \%$ & $7.00 \%$ & $7.00 \%$ & $7.00 \%$ & $7.00 \%$ & $7.00 \%$ \\
\hline & EMB + & 137 & 137 & 137 & 137 & 137 & 137 & 137 & 137 & 137 & 137 & 10 \\
\hline & Costo Deuda & $11.02 \%$ & $11.22 \%$ & $11.84 \%$ & $1207 \%$ & $1252 \%$ & $12.97 \%$ & $1207 \%$ & $1207 \%$ & $12.07 \%$ & $12.07 \%$ & $1207 \%$ \\
\hline & Costodesp mp & $11.015 \%$ & $11.22 \%$ & $11.84 \%$ & $1207 \%$ & $1252 \%$ & $12.97 \%$ & $1207 \%$ & $1207 \%$ & $12.07 \%$ & $12.07 \%$ & $1207 \%$ \\
\hline & Costo capta (Ks) & 0.1141 & $11.46 \%$ & $11.45 \%$ & $11.56 \%$ & $11.75 \%$ & $11.75 \%$ & $13.12 \%$ & $14.49 \%$ & $15.88 \%$ & $1723 \%$ & $1860 \%$ \\
\hline & Tasa di mpesstos & $0 \%$ & $0 \%$ & $0 \%$ & $0 \%$ & $\%$ & $0 \%$ & $0 \%$ & $0 \%$ & $0 \%$ & $0 \%$ & \\
\hline
\end{tabular}

Cuadro E. Cálculo de la Tasa de Descuento

\begin{tabular}{|c|c|c|c|c|c|c|c|c|c|c|c|c|}
\hline & 2009 & 2010 & 2011 & 2012 & 2013 & 2014 & 2015 & 2016 & 2017 & 2018 & 2019 & 2020 \\
\hline Deuda con costo & 631,829 & 690.829 & 789.977 & 884,038 & 970.927 & $1,061,988$ & $1,157,179$ & $1,118.823$ & 1,0000000 & 1,035948 & 976,372 & 916.198 \\
\hline Capital & .68 .840 & 7.687 & 58,102 & 137,988 & 259024 & 381,187 & 528.984 & 717,576 & 968388 & 1.184 .274 & 1,438897 & 1.712415 \\
\hline Devda Nabr de la empresa & $112 \%$ & $99 \%$ & $93 \%$ & $87 \%$ & $79 \%$ & $74 \%$ & $69 \%$ & $61 \%$ & $53 \%$ & $47 \%$ & $40 \%$ & $35 \%$ \\
\hline Coptel Wabros a emperse & $.1180 \%$ & $1.10 \%$ & $0.30 \%$ & $1350 \%$ & $21.00 \%$ & $20.41 \%$ & $31.37 \%$ & $3906 \%$ & $4702 \%$ & $5334 \%$ & $39.57 \%$ & $60.15 \%$ \\
\hline wacc & $1097 \%$ & $11.02 \%$ & $1124 \%$ & $11.62 \%$ & $11.96 \%$ & $1232 \%$ & 125936 & $1248 \%$ & $1321 \%$ & $14.10 \%$ & $15.15 \%$ & $16.33 \%$ \\
\hline
\end{tabular}


Cuadro F. Cálculo de Flujos Descontados

\begin{tabular}{|c|c|c|c|c|c|c|c|c|c|c|c|c|}
\hline & $\begin{array}{r}2010 \\
181003\end{array}$ & $\begin{array}{r}2011 \\
151,521\end{array}$ & $\begin{array}{r}2012 \\
140400\end{array}$ & $\begin{array}{r}2013 \\
170221\end{array}$ & $\begin{array}{r}2014 \\
1770532\end{array}$ & $\begin{array}{r}2015 \\
20079\end{array}$ & $\begin{array}{r}2016 \\
239278\end{array}$ & $\begin{array}{r}2017 \\
290507\end{array}$ & $\begin{array}{r}2018 \\
275856\end{array}$ & $\begin{array}{r}2019 \\
303987\end{array}$ & $\begin{array}{r}2020 \\
323469\end{array}$ & $\begin{array}{l}\text { Perpetudad } \\
3.137 .189\end{array}$ \\
\hline 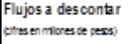 & 161,003 & 151.521 & 140.400 & 170.221 & 177.052 & 202,779 & 239.278 & 290.507 & 275,565 & 303.987 & 323,469 & $3,137,189$ \\
\hline 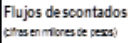 & 145,503 & 122.450 & 100.969 & 108.315 & 99.040 & 99.570 & 104.358 & 112.538 & 94.817 & 92.903 & 87.007 & 756.403 \\
\hline
\end{tabular}

Escenario Dos: Suponiendo que los derechos (regalías) al gobierno, fueran la mitad que las que se pagan bajo el escenario base.

Resultado de la Valuaciòn Escenario: Reducción Derechos (Regalias) en 50\%

\begin{tabular}{|l|rr|}
\hline & Mill. Pesos & Mill. Dòlares \\
\hline \hline Suma de Flujos Descontados & $3,363,171$ & 265,063 \\
Perpetuidad Descontada & $2,107,775$ & 166,121 \\
Valor de los activos & $5,470,946$ & $\mathbf{4 3 1 , 1 8 4}$ \\
Deuda con Costo al 30 sep. 2010 & 652,828 & $\mathbf{5 1 , 4 5 2}$ \\
Valor de la empresa & $4,818,118$ & \\
Valor de la empresa / Deuda con Costo & 7.38 & \\
Apalancamiento (deuda con costo / Valor de la empresa) & 0.14 & \\
Tipo de cambio peso / dólar al 30 Sep. 2010 & & 12.6882 \\
\hline
\end{tabular}

La valuación resultante en este escenario presenta un incremento muy significativo de casi 6 veces respecto al escenario base, por dos razones principales:

La empresa genera utilidades de poco más de la mitad de la utilidad operativa con su respectivo impacto positivo en el capital contable de Pemex.

Parte de flujo generado lo canalicé al pago de deuda con costo por lo que el monto de intereses pagados disminuye asimismo el excedente de efectivo genera intereses; el efecto combinado de estos dos aspectos contribuye a la generación de utilidad neta desde el inicio del período de estimación.

Con esta valuación, Pemex cubre su deuda con costo en los primeros dos años de estimación por lo que bajo este escenario suponemos que las inversiones se financian con recursos propios. La razón de apalancamiento es del orden del $14 \%$. Se observa en este escenario que el capital contable pondera de manera muy importante en este escenario por lo que la tasa de descuento se incrementa casi tres veces más respecto al escenario base; sin embargo no tiene un impacto significativo en la valuación ya que los flujos crecen en mayor proporción. Los cuadros G, H e I muestran la valuación en este escenario, así como los cuadros con los cálculos del Costo de Capital, la Tasa de Descuento y los Flujos Descontados y sus supuestos.

Cuadros G, H e I: Cálculos del Costo de Capital (Ks), WACC y flujos descontados del Escenario Dos:

Cuadro G. Cálculo del $K_{s}$ a tráves del CAPM

\begin{tabular}{|c|c|c|c|c|c|c|c|c|c|c|c|c|}
\hline \multirow[t]{7}{*}{ Buboustos } & & 2010 & 2011 & 2012 & 2013 & 2014 & 2015 & 2016 & 2017 & 2018 & 2019 & \\
\hline & Bote & 3.47 & 3.47 & 3.47 & 3.47 & 3.47 & 3.47 & 4.47 & 5.47 & 6.47 & 7.47 & \\
\hline & Bono 10 mos posos & $6.66 \%$ & $6.70 \%$ & $6.70 \%$ & $6.00 \%$ & $7.00 \%$ & $7.00 \%$ & $7.00 \%$ & $7.00 \%$ & $700 \%$ & $700 \%$ & $7.00 \%$ \\
\hline & & 137 & 137 & 137 & 137 & 137 & 137 & 137 & 137 & 137 & & 137 \\
\hline & Cosso Douss & $11.02 \%$ & $11.22 \%$ & $11.64 \%$ & $12.07 \%$ & $12.52 \%$ & $12.97 \%$ & $1297 \%$ & $1297 \%$ & $1297 \%$ & $1297 \%$ & $1297 \%$ \\
\hline & $\cos 50 \cos s .1 m \theta$ & $11.015 \%$ & $11.22 \%$ & $11.64 \%$ & $12.07 \%$ & $12.52 \%$ & $12.97 \%$ & $1297 \%$ & $1297 \%$ & $1297 \%$ & $1297 \%$ & $1297 \%$ \\
\hline & 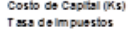 & 0.1141 & $11.45 \%$ & $11.45 \%$ & $11.55 \%$ & $11.75 \%$ & $11.75 \%$ & $13.12 \%$ & $14.49 \%$ & $15.86 \%$ & $1723 \%$ & $1860 \% /$ \\
\hline
\end{tabular}


Cuadro H. Cálculo de la Tasa de Descuento

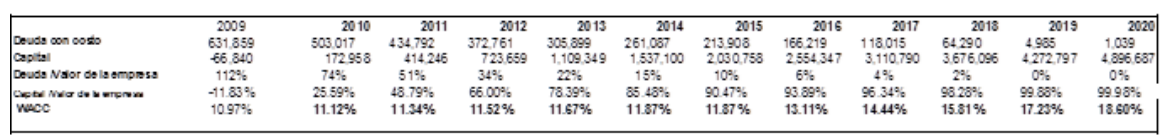

Cuadro I. Cálculo de Flujos Descontados

\begin{tabular}{|c|c|c|c|c|c|c|c|c|c|c|c|c|}
\hline Flujos a dascontar & $\begin{array}{r}2010 \\
449.315\end{array}$ & $\begin{array}{r}2011 \\
461,700\end{array}$ & $\begin{array}{r}2012 \\
4885545\end{array}$ & $\begin{array}{r}2013 \\
361.326\end{array}$ & $\begin{array}{r}2014 \\
614,337\end{array}$ & $\begin{array}{r}2015 \\
684686\end{array}$ & $\begin{array}{r}2016 \\
715208\end{array}$ & $\begin{array}{r}2017 \\
751781\end{array}$ & $\begin{array}{r}2018 \\
705695\end{array}$ & $\begin{array}{r}2019 \\
202327\end{array}$ & $\begin{array}{r}2020 \\
832831\end{array}$ & Forpotudeds \\
\hline 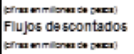 & 404,360 & 372,468 & 352269 & 361.015 & 350,690 & 349,321 & 326,177 & 306,430 & 279.033 & 261,359 & 243.124 & $2,107,775$ \\
\hline
\end{tabular}

Escenario Tres: Determinación de un precio del petróleo que provoca una situación de equilibrio en la generación de FNO. Ejemplo cuando el precio internacional del petróleo es de US\$52.52 dólares por barril.

Resultado de la Valuaciòn Escenario Base

\begin{tabular}{|l|rr|}
\hline \hline & Mill. Pesos & Mill. Dòlares \\
\hline \hline Suma de Flujos Descontados & 115,161 & 9,076 \\
Perpetuidad Descontada & 537,226 & 42,341 \\
Valor de los activos & $\mathbf{6 5 2 , 3 8 7}$ & $\mathbf{5 1 , 4 1 7}$ \\
Deuda con Costo al 30 sep. 2010 & $\mathbf{6 5 2 , 8 2 8}$ & $\mathbf{5 1 , 4 5 2}$ \\
Valor de la empresa & $\mathbf{- 4 4 1}$ & \\
Valor de la empresa / Deuda con Costo & 0.00 \\
Apalancamiento (deuda con costo / Valor de la empresa) & -1481.50 \\
Tipo de cambio peso / dólar al 30 Sep. 2010 & \multicolumn{2}{|c|}{12.6882} \\
\hline
\end{tabular}

El tercer escenario presenta un valor de la empresa de prácticamente cero como se observa en el Cuadro. Lo atribuimos esto a que a un precio de US\$52.52 por barril los ingresos estimados crecen a menor ritmo, en promedio $6.0 \%$ vs el $7.5 \%$ del escenario base. Este menor crecimiento de los ingresos, aunado al efecto de la reserva laboral sobre los costos y gastos provocan la disminución de la utilidad de operación en un $9.0 \%$ en promedio, comparada con la estimada para el escenario base. El resultado neto de este escenario se caracteriza por pérdidas a lo largo del período de estimación. Esta pérdida neta se refleja directamente en el Capital Contable, haciéndolo más negativo respecto al que estimé en el escenario base. El flujo operativo crece a menor ritmo, bajo el supuesto de este escenario, provocando que Pemex difícilmente puede financiar sus inversiones de capital asimismo cubrir sus pasivos con costo. La combinación de los factores que acabo de mencionar así como la ponderación de la deuda se reflejan en una tasa de descuento de casi el doble de la utilizada en el escenario base, afectando negativamente la valuación de la empresa. Los cuadros J, K y L muestran los cálculos del costo de capital y la tasa de descuento utilizados en este escenario. 
Cuadros J, K y L: Cálculos del Costo de Capital (Ks), WACC y flujos descontados del Escenario Tres:

Cuadro J. Cálculo del $K_{s}$ a tráves del CAPM

\begin{tabular}{|c|c|c|c|c|c|c|c|c|c|c|c|c|}
\hline Budusstos & 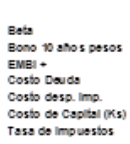 & $\begin{array}{r}2010 \\
3.47 \\
666 \% \\
117 \\
11.02 \% \\
11.015 \% \\
0.1114 \\
0 \%\end{array}$ & $\begin{array}{r}2011 \\
3.47 \\
6.70 \% \\
137 \\
1122 \% \\
1122 \% \\
11.45 \% \\
0 \%\end{array}$ & $\begin{array}{r}2012 \\
3.47 \\
6.70 \% \\
137 \\
11.64 \% \\
11.64 \% \\
11.45 \% \\
0 \%\end{array}$ & $\begin{array}{r}2013 \\
3.47 \\
6.50 \% \\
137 \\
12.07 \% \\
12.07 \% \\
11.55 \% \\
0 \%\end{array}$ & $\begin{array}{r}2014 \\
3.47 \\
7.00 \% \\
137 \\
12.52 \% \\
12.52 \% \\
11.75 \% \\
0 \%\end{array}$ & $\begin{array}{r}2015 \\
3.47 \\
7.00 \% \\
137 \\
12.97 \% \\
12.97 \% \\
11.75 \% \\
0 \%\end{array}$ & $\begin{array}{r}2016 \\
4.47 \\
7.00 \% \\
137 \\
12.97 \% \\
12.9 \% \\
13.12 \% \\
0 \%\end{array}$ & $\begin{array}{r}2017 \\
5.47 \\
7.00 \% \\
137 \\
1297 \% \\
1297 \% \\
14.99 \% \\
0 \%\end{array}$ & $\begin{array}{r}2018 \\
6.47 \\
7.00 \% \\
137 \\
12.97 \% \\
1297 \% \\
15.86 \% \\
0 \%\end{array}$ & $\begin{array}{r}2019 \\
7.47 \\
7.00 \% \\
137 \\
1297 \% \\
1297 \% \\
17.23 \% \\
0 \%\end{array}$ & 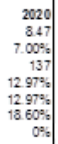 \\
\hline
\end{tabular}

Cuadro K. Cálculo de la Tasa de Descuento

\begin{tabular}{|c|c|c|c|c|c|c|c|c|c|c|c|c|}
\hline & 2009 & 2010 & 2011 & 2012 & 2013 & 2014 & 2015 & 2016 & 2017 & 2018 & 2019 & 2020 \\
\hline Devda con costo & 631.859 & 690.829 & 789.977 & 884,038 & 970.927 & 1.061 .965 & $1,157,179$ & 1.239 .682 & 1323077 & $1,407.372$ & 1.492 .578 & 1.578705 \\
\hline Capital & 86.840 & -276.823 & 532341 & -775.089 & $-1,000620$ & $-1,247,798$ & $-1,492123$ & $-1,528757$ & $-1,548,121$ & $-1,800342$ & -1.056 .980 & $-1,724,475$ \\
\hline Deuda Nator de la emplasa & $112 \%$ & $167 \%$ & $307 \%$ & $811 \%$ & $-3270 \%$ & $-571 \%$ & $.345 \%$ & $-429 \%$ & $288 \%$ & $-729 \%$ & $-814 \%$ & $-1083 \%$ \\
\hline Captei/Nair co is empress & $-11.83 \%$ & $8680 \%$ & $-20663 \%$ & $711.42 \%$ & $3369.91 \%$ & $671.43 \%$ & $445.49 \%$ & $528.84 \%$ & $687.92 \%$ & $829.32 \%$ & $1013.55 \%$ & $118301 \%$ \\
\hline WaCC & $1097 \%$ & $10.75 \%$ & $10.75 \%$ & $1297 \%$ & $5.45 \%$ & $7.35 \%$ & $7.57 \%$ & $13.80 \%$ & $23.48 \%$ & $37.00 \%$ & $56.23 \%$ & $79.67 \%$ \\
\hline
\end{tabular}

Cuadro L. Cálculo de Flujos Descontados

\begin{tabular}{|c|c|c|c|c|c|c|c|c|c|c|c|c|}
\hline & 2010 & 2011 & 2012 & 2013 & 2014 & 2015 & 2016 & 2017 & 2018 & 2019 & 2020 & Perpetididad \\
\hline 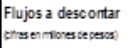 & 44.001 & $-40,372$ & -26.331 & -53.741 & 86.700 & 80714 & 124,491 & 166.242 & 138480 & 140.361 & 132933 & 1.289 .259 \\
\hline $\begin{array}{l}\text { Flujos des contedos } \\
\text { enturnerestesesas }\end{array}$ & 39.730 & -32.916 & -45.309 & -87.285 & 46.815 & 39.152 & 74,707 & 92744 & 71.821 & 67.875 & 59.584 & 537.226 \\
\hline
\end{tabular}

\section{2) Aplicación práctica del Modelo Binomial en Pemex:}

Para valuar una opción a través de un Modelo Binomial se requiere una hoja de cálculo de Excel para calcular el valor del bono, basada en los flujos libres de Pemex. Se calculan previamente los siguientes valores: $u=e^{\operatorname{raìz~de}(\delta t)}, d=1 / u, p=\left(e^{u(\delta t)}-d\right) /(u-d)$

La manera en que las opciones son valuadas en el modelo binomial, es empezando desde el último nodo del árbol binomial (es decir en el tiempo $T$ ) y regresando del final hacia el principio del árbol.

El cuadro M muestra el valor de Pemex, bajo diferentes escenarios. La idea es valuar el precio de una opción vinculada a los bonos emitidos por el VPE.

Cuadro M: Valor de Pemex bajo los diferentes escenarios

\begin{tabular}{|l|l|c|}
\hline & & $\begin{array}{c}\text { Valor del Bono=Valor de Empresa (1) } \\
\text { (Miles de millones de pesos, mmp) }\end{array}$ \\
\hline Escenario Base & $\begin{array}{l}\text { Situación financiera y operativa actual de } \\
\text { Pemex, a un precio del petróleo de } \\
\text { US\$74 por barril }\end{array}$ & 765 \\
\hline Escenario Uno & $\begin{array}{l}\text { Escenario Base, eliminando pasivos } \\
\text { laborales }\end{array}$ & 1,184 \\
\hline Escenario Dos & $\begin{array}{l}\text { Escenario Base, reduciendo en 50\% la } \\
\text { tasa de regalías (impuestos y derechos) }\end{array}$ & 4,818 \\
\hline Escenario Equilibrio & $\begin{array}{l}\text { Escenario Base calculado a un precio del } \\
\text { petróleo de US\$50 por barril }\end{array}$ & 0 \\
\hline
\end{tabular}

(1) Valor de la Empresa: Valor presente de los FNO menos Pasivos con Costo 
122 Nueva Época REMEF (The Mexican Journal of Economics and Finance)

\section{Ejercicio 1 para Valuar Pemex:}

Suponemos una opción de compra que deberá ejercer la Alta Gerencia de Pemex, para comprar flujos adicionales de efectivo si se cumplen las siguientes condiciones:

Si el valor de la empresa bajo el Escenario Base es de MXN\$765 mmp. y si lo comparamos con el valor de la empresa en el Escenario Uno por MXN\$1,184 mmp. (donde se eliminan los pasivos laborales), obtenemos una diferencia entre ambos valores de MXN\$419 mmp. Es decir, que Pemex tendría un valor superior en $55 \%$ al Escenario Base, debido a una mayor retención de flujos operativos en la propia empresa, ya que se evitaría el pago presente y futuro de sus obligaciones laborales (jubilaciones).

Para poder deshacer los pasivos laborales, podemos suponer que la empresa debería pagar al sindicato una compensación, que costaría a Pemex MXN\$800 millones. De acuerdo a lo anterior, procederé a construir un árbol binomial partiendo de un nodo inicial con valor de $\mathrm{MXN} \$ 419 \mathrm{mmp}$, para calcular el valor de la opción por estos flujos adicionales. Suponemos en este ejemplo que no se pagan dividendos (regalías).

A continuación se presentan las variables utilizadas para alimentar la hoja de cálculo:

\begin{tabular}{|c|c|}
\hline$S_{o}$ Valor actual & MXN \$419 mmp \\
\hline$S_{t}$ Precio de Ejercicio & MXN\$ $800 \mathrm{mmp}$ \\
\hline Volatilidad precio del petróleo (anual) & $25.5 \%$ \\
\hline Plazo para ejercer la opción & 5 años \\
\hline Tipo de opción & Americana \\
\hline Ramas del árbol & 10 \\
\hline
\end{tabular}

El árbol binomial resultante, en la Figura 6, muestra que es necesario esperar hasta el final de la vida de la opción, para que convenga ejercerla; es decir, cuando aparecen los valores señalados con flechas. El primer renglón de cada nodo muestra el valor futuro de la empresa y el segundo renglón el valor de la opción). 
Figura 4: Árbol binomial del valor de la opción en Ejercicio 1.

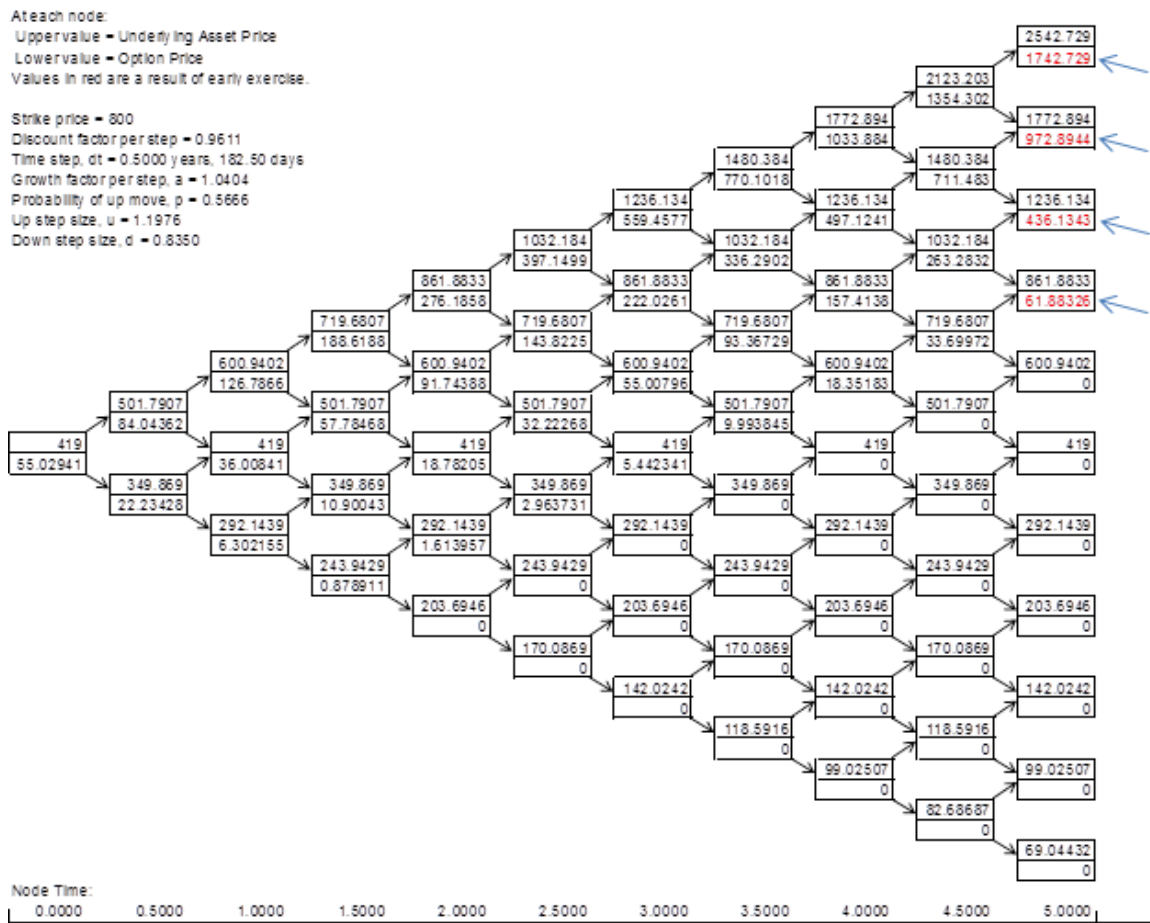

En cada nodo, el valor superior $=$ precio del subyacente, el valor inferior $=$ precio de la opción, precio de ejercicio $=730$, factor de descuento por paso $=0.9611$, paso en el tiempo, $d_{t}=0.5$ años, factor de crecimiento por paso $a=0.7259$, probabilidad de moverse hacia arriba $=0.3009$, tamaño del paso hacia arriba $u=1.1976$, tamaño del paso hacia abajo $d=0.8350$. Sólo en el nodo inferior derecho se ejerce la opción.

En este caso el valor de Pemex en el nodo cero (to) es de MXN\$765 mmp (el valor presente de los FNO en el Escenario Base, que como dije es el valor del bono, o de la empresa), más el valor de la opción de MXN\$55 mmp (primer nodo de izquierda a derecha de Figura 4), es decir que el valor total de Pemex es de MXN\$820 mmp.

\section{Ejercicio 2 para valuar Pemex:}

Suponemos una opción de compra que deberá ejercer la Alta Gerencia de Pemex, para comprar flujos adicionales de efectivo, bajo las mismas condiciones que el ejercicio 1, pero suponiendo que los pagos de regalías son equivalentes a pago de dividendos:

Valuamos esta opción de compra sobre un activo que paga dividendos. La tasa de dividendos es de $72 \%$ que resulta de dividir las regalías (impuestos y 
124 Nueva Época REMEF (The Mexican Journal of Economics and Finance)

derechos) de un año, que en 2010 fueron por MXN\$554 mmp. entre el valor de la empresa en el escenario base de MXN\$765 mmp. El árbol binomial resultante (Figura 7) muestra que la opción puede ejercerse a partir del año 4. El siguiente árbol binomial se construyó bajo los mismos supuestos que el ejercicio 1, pero seleccionamos el pago de dividendos, bajo una estructura binomial americana.

Figura 5: Valuación de la opción suponiendo que el pago de regalías es igual a los dividendos:

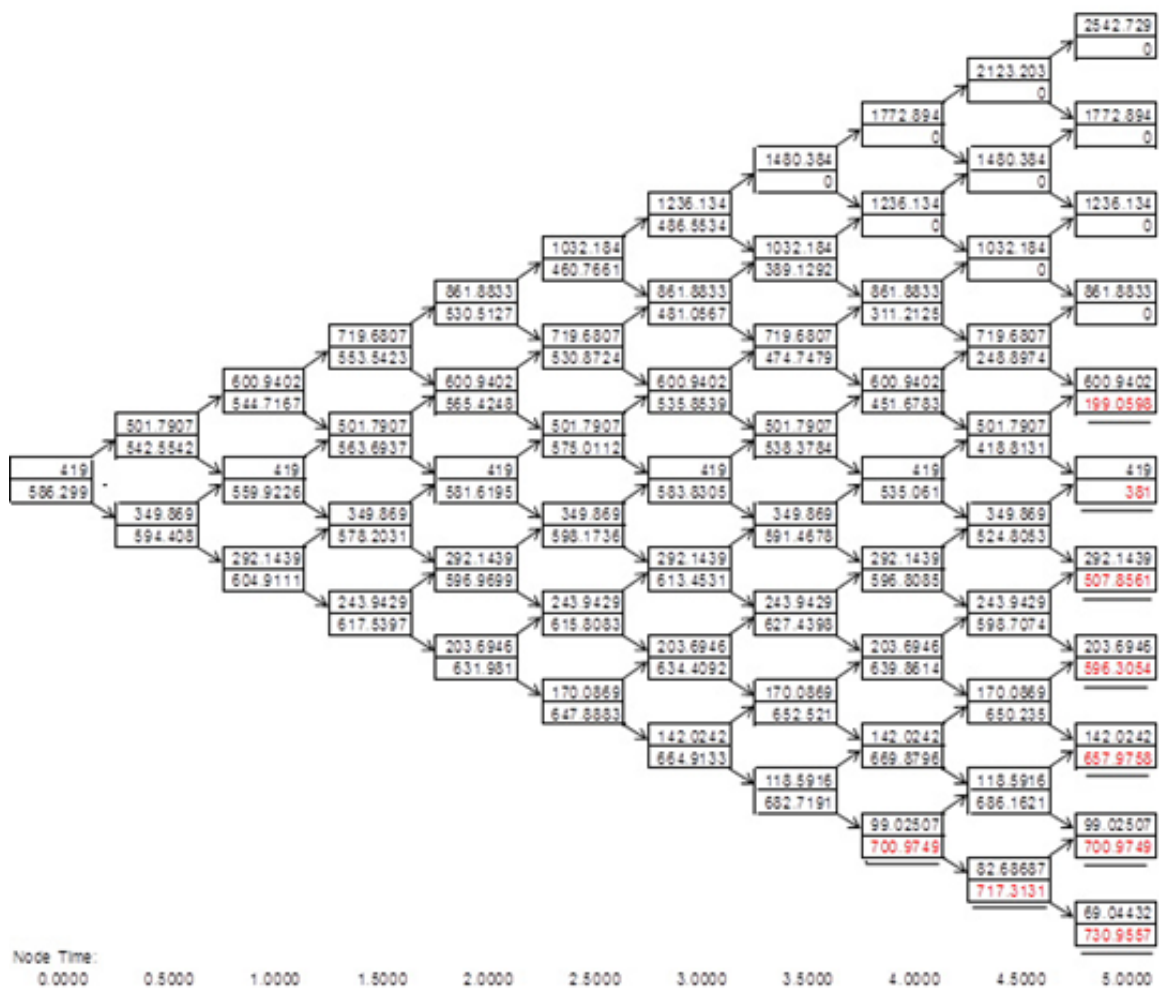

En cada nodo, el valor superior $=$ precio del subyacente, el valor inferior $=$ precio de la opción, precio de ejercicio $=730$, factor de descuento por paso $=0.9611$, paso en el tiempo, $d_{t}=0.5$ años, factor de crecimiento por paso $a=0.7259$, probabilidad de moverse hacia arriba $=0.3009$, tamaño del paso hacia arriba $u=1.1976$, tamaño del paso hacia abajo $d=0.8350$. Sólo en el nodo inferior derecho se ejerce la opción.

En este caso el valor de Pemex en el nodo cero (to) es de MXN\$765 mmp (el valor presente de los FNO en el Escenario Base, que como dije es el valor del bono, o de la empresa), más el valor de la opción de MXN\$586 mmp (primer nodo de izquierda a derecha de Figura 5), es decir que el valor total de Pemex es de MXN\$1,351 mmp.

El mayor valor de Pemex se genera por el pago de dividendos (o regalías) 
Visto de otra forma, podemos suponer que el valor inicial del árbol binominal es MXN $\$ 4,053 \mathrm{mmp}$, que es la diferencia entre el valor de Pemex de MXN\$4,818 (Escenario Dos, cuando se hace el supuesto de disminuir la tasa de regalías en $50 \%$, desde $72 \%$ hasta $35.5 \%$ ) y el valor de Pemex en el Escenario Base de MXN\$765 mmp, es decir MXN\$4,818 mmp - MXN\$765 mmp $=$ MXN $\$ 4,053 \mathrm{mmp}$.

Figura 8: Valuación de la opción con valor inicial de MXN\$4,053 millones: de regalías es igual a los dividendos:

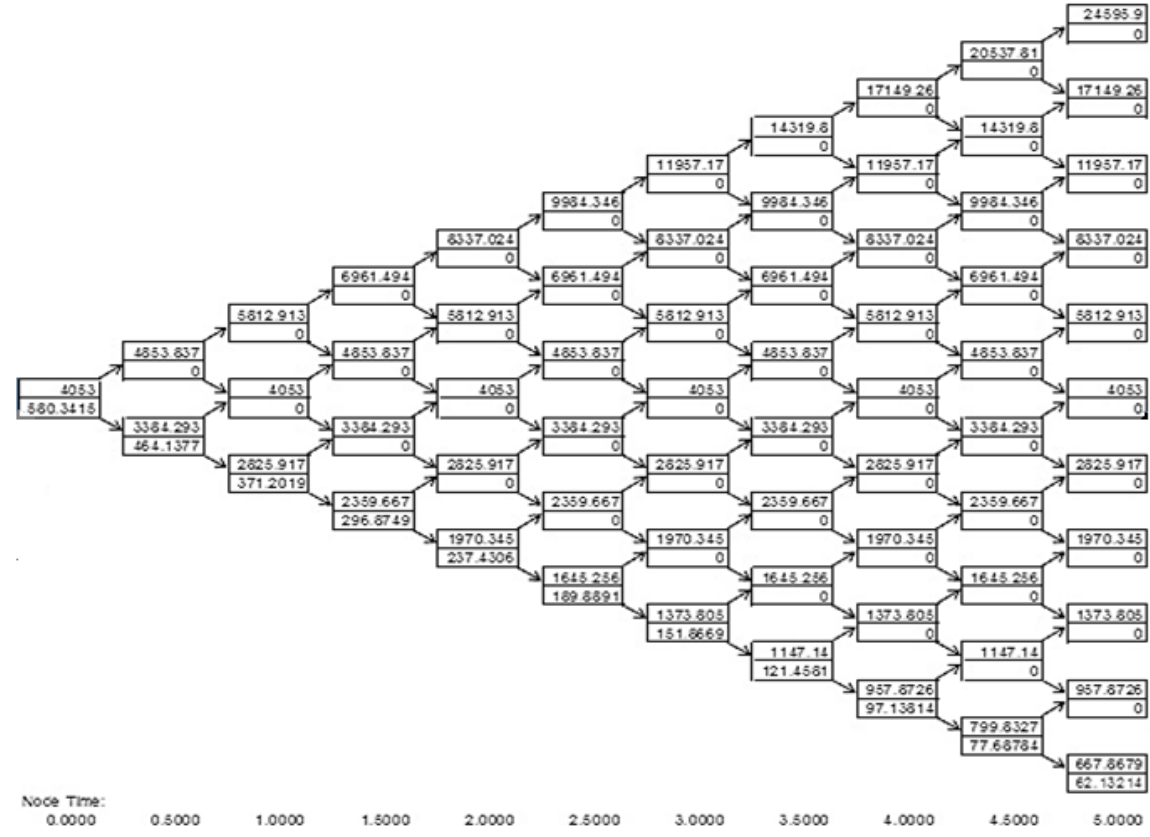

En cada nodo, el valor superior $=$ precio del subyacente, el valor inferior $=$ precio de la opción, precio de ejercicio $=730$, factor de descuento por paso $=0.9611$, paso en el tiempo, $d_{t}=0.5$ años, factor de crecimiento por paso $a=0.7259$, probabilidad de moverse hacia arriba $=0.3009$, tamaño del paso hacia arriba $u=1.1976$, tamaño del paso hacia abajo $d=0.8350$. Sólo en el nodo inferior derecho se ejerce la opción.

En este último ejercicio, el valor de la opción es de MXN\$580 mmp, que sumados al valor presente de los FNO de Pemex bajo el Escenario Base de MXN $\$ 765 \mathrm{mmp}$, nos arroja un valor total de la empresa actual de MXN\$1,345 mmp. En este ejercicio la opción se podrá ejercer al final de los 5 años. El mayor valor de Pemex se genera por el pago de dividendos (o regalías) 


\section{Conclusiones}

Consideramos que la aportación más relevante es que esta propuesta de valuación de empresas estratégicas descentralizadas que exploran y explotan recursos naturales, como un bono estructurado a través de un "Vehículo de Propósito Especial", permite ver a Pemex como un mecanismo flexible (sin violar la soberanía de la nación sobre la propiedad de recursos naturales) que utiliza el Gobierno para explorar y explotar a los mismos, y por lo tanto puede ser sujeto a diferentes formas de financiamiento, incluyendo la emisión de acciones al gran público inversionista, que mejoraría la estructura de capital y aumentaría su valuación como empresa, o al ser vista como un VPE se incrementaría la valuación del mismo.

También Pemex puede ser visto o manejado como un Vehículo de Propósito Especial Maestro, del cual pueden colgar otros Vehículos de Propósito Especial más particulares, relacionados con las subsidiarias de Pemex (Exploración, Petroquímica, Gas etc.) que permitan ofrecer bonos especializados por cada negocio y con un riesgo de mercado diferente. El VPE Maestro puede diseñarse para minimizar el riesgo de todos los bonos que se emitan a través de este, haciendo un "pool" con todos los recursos líquidos que genere el VPE y puedan ser usados para hacer pagos a los diferentes tenedores de bonos.

La valuación de Pemex bajo métodos tradicionales de flujos descontados (DCF, por sus siglas en inglés), y de acuerdo a diferentes escenarios nos permite concluir, que esta empresa siempre tienen un valor presente positivo en el valor de sus flujos operativos (una vez que se ha cubierto deuda con costo), siempre que el precio del petróleo se ubique por arriba de US\$50 por barril.

Cuando el Escenario Base (bajo condiciones actuales de Pemex) se calcula con un precio de petróleo menor a US\$50 por barril, la empresa podría compensar el valor presente de sus flujos, a través de medidas que por el momento son teóricas, tales como la reducción en el pago de regalías, la reestructura o eliminación de sus pasivos labores, etc.

En los ejercicios de valuación de Pemex visto como un bono estructurado, que incluye opciones para la empresa, para que pueda aumentar el monto de sus flujos de operación, podemos observar que el valor de la empresa obtenido por DCF se puede mejorar, si la empresa incluye como opciones la reducción en el pago de regalías, y/o con la eliminación de pasivos laborales.

El escenario con mayor valor para la empresa es aquel donde se contempla una tasa de pago de dividendos de $72 \%$ (equivalente a la tasa de pago de regalías), concluyendo que el activo más valioso de la empresa es la generación de flujo de efectivo de operación.

Por lo tanto, dejar de ver a Pemex y a otras empresas dedicadas a la exploración y explotación de recursos naturales (que no son de su propiedad y cuyo precio corresponde al de precios internacionales de materias primas o energéticos) como empresas tradicionales y valuarlas como bonos estructurados, es una herramienta que permite conocer su valor en forma más objetiva. 


\section{Bibliografía}

Brigham, E. F. and J. F. Houston (1998). Fundamentals of Financial Management, 8a. ed. Constitución Política de los Estados Unidos Mexicanos (1917). Diario Oficial de la Federación, modificada a julio 14, 2011.

Fabozzi, F. J. (2000). Fixed Income Analysis for the CFA Program, Frank J. Fabozzi Associates, New Hope, Pennsylvania.

Fabozzi, F. J., H. A. Davis, M. Choudhry (2006). Introduction to Structured Finance, Willey.

Financial Accounting Standards Board of the Financial Accounting Foundation, (2010). Overview of Oil and Gas Accounting \& PSC Accounting, Global and Gas Center, Financial Accounting Series, Ernst \& Young, ISSN-0885.9051

Hull, J. C. (2000). Options, Futures and Other Derivative Securities, Prentice Hall , 2a edición.

Koller, T., M. Goedhart and D. Wessels (2010). Valuation: Measuring and Managing the Value of Companies, McKinsey and Co

Kogan, J., A. Espinosa and O. Zelaya (2011). Rocks for jocks...a Wall St. guide to Pemex. The Emerging Markets Slapshot, Scotia Capital, April 20th

Kroll, K. (1998). Options Theory applied to Business Decisions, Industry Week, febrero.

Ley del Impuesto sobre la Renta (2009). Diario Oficial de la Federación, modificada a diciembre 31, 2010.

Ley Federal de Derechos (1988). Diario Oficial de la Federación, modificada a noviembre 18, 2011.

Lintner, J. (1965). The Valuation of Risk Assets and the Selection of Risky Investments in Stock Portfolios and Capital Budgets, Review of Economics and Statistics, Febrero, pp. 13-37.

Markowitz, H. M. (1959). Portfolio Selection: Efficient diversification of Investment, Cowles Foundation Monograph 16, Yale University Press, New Haven.

Proyectos estratégicos de Pemex (2011), Pemex Panorama, marzo, p. 12.

Reporte de Resultados de Petróleos Mexicanos, Organismos Subsidiarios y Compañías Subsidiarias al 31 de marzo de 2011 [Reporte Pemex] (2011, 4 de mayo).

Reservas probadas al 1 enero de 2011 de Pemex al 30 de marzo 2010 en www.pemex.com.

Sharpe, W. (1964). Capital Asset Prices: A Theory of Market Equilibrium under Conditions of Risk, Management Science, pp. 277-293.

Tasa de restitución de reservas de Pemex (2012)

Tobin, J. (1958). Liquidity Preference as behavior Towards Risk, Review of Economic Studies, febrero, pp. 65-86.

Todo sobre la reforma energética, Primer decreto: Cambios en el régimen fiscal de Pemex (2011) de www.pemex.com al 27 de julio de 2011

Valencia-Herrera, H. (2009). Mejores prácticas de inversión, Mejores prácticas financieras de las empresas en México, IMEF. 